
allemande

46-1 | 2014

Les fondements normatifs de l'État constitutionnel moderne en Allemagne. Une approche pluridisciplinaire

\title{
La notion « d'ordre étatique » dans les manuels scolaires de la République de Weimar
}

\author{
Marie-Bénédicte Vincent
}

\section{OpenEdition}

Journals

Édition électronique

URL : https://journals.openedition.org/allemagne/1264

DOI : $10.4000 /$ allemagne. 1264

ISSN : 2605-7913

Éditeur

Société d'études allemandes

Édition imprimée

Date de publication : 30 juin 2014

Pagination : 63-81

ISSN : 0035-0974

Référence électronique

Marie-Bénédicte Vincent, «La notion « d'ordre étatique » dans les manuels scolaires de la République de Weimar », Revue d'Allemagne et des pays de langue allemande [En ligne], 46-1 | 2014, mis en ligne le 29 juillet 2019, consulté le 18 mai 2021. URL : http://journals.openedition.org/allemagne/1264 ; DOI https://doi.org/10.4000/allemagne.1264 


\title{
La notion « d'ordre étatique » dans les manuels scolaires de la République de Weimar
}

\author{
- Marie-Bénédicte Vincent*
}

\section{Introduction}

La notion «d'ordre étatique» (Staatsordnung) est un terme fréquemment utilisé dans les manuels scolaires d'histoire et d'éducation civique produits sous la République de Weimar. Elle inclut bien sûr l'ensemble des institutions du régime républicain, mais est associée aussi à des valeurs politiques plus larges: celles-ci intéressent l'historien car elles montrent comment est perçu à l'époque le nouveau régime au regard de l'histoire allemande récente (Kaiserreich et césure de 1918) ou plus ancienne (révolution de 1848, unité de 1871). C'est donc à une histoire des représentations de l'État qu'invite la lecture des manuels scolaires, un État dont la forme démocratique est hautement controversée après la révolution de 1918. C’est pourquoi les républicains, soucieux de consolider le régime et susciter chez les jeunes générations une adhésion à la démocratie, ont inscrit dans la Constitution du 11 août 1919 l'obligation d'une éducation civique (Staatsbürgerkunde), ainsi que la délivrance à tous les élèves en fin de scolarité d'un exemplaire de la Constitution (article $148 \$ 3$ ). Cette prescription explique la floraison de manuels d'éducation civique à partir de $1919^{(1)}$.

Les études sur la culture scolaire allemande se multiplient depuis quelques années, notamment celles adoptant une démarche comparative ${ }^{(2)}$ ou portant sur le $\mathrm{xx}^{\mathrm{e}}$ siècle

* Maître de conférences en histoire contemporaine, ENS UIm, membre de l'Institut d'histoire moderne et contemporaine (IHMC).

1 Ceux-ci ont pour le moment été relativement peu étudiés. Une étude de Karin Kitowski porte sur trois d'entre deux: "'Ein warmer Hauch der Liebe zu Volk und Staat...' Anspruch und Wirklichkeit der Staatsbürgerkunde in der Weimarer Republik », in: Karin Kiтowsкi et Rüdiger Wulf (éd.), Die Liebe zu Volk und Vaterland. Erziehung zum Staatsbürger in der Weimarer Republik, Dortmund, Westfälisches Schulmuseum, 2000, p. 9-30.

2 Jeannie Bauvois-Cauchepin, Enseignement de l'histoire et mythologie nationale: Allemagne-France du début du xx siècle aux années 1950, Berne, Peter Lang, 2002; Maria Christina Giuntella, "Enseignement de l'histoire et révision des manuels scolaires dans l'entre-deux-guerres », in: Marie-Christine 
récent ${ }^{(3)}$. Elles croisent histoire politique et histoire culturelle, en s'intéressant à la transmission des savoirs et des contenus idéologiques, aux médiateurs que sont les enseignants et enfin aux enjeux de la réception par les jeunes. L'analyse des manuels scolaires s'inscrit dans cette dynamique: elle fait l'objet d'une méthodologie spécifique $^{(4)}$. Le corpus étudié se compose de trente-quatre manuels d'éducation civique et trente manuels d'histoire édités sous la République de Weimar et conservés au Georg-Institut für Internationale Schulbuchforschung de Brunswick (voir leur présentation en annexe). La réflexion qui suit s'appuie sur l'ensemble de ces titres, même si seules quelques citations ont été restituées. Les manuels examinés correspondent à différents niveaux scolaires. La Constitution de Weimar prévoit une obligation scolaire de huit ans dont quatre au sein d'une Grundschule (art. 146-1), les élèves se partageant ensuite entre Volksschule de la $5^{\mathrm{e}}$ à la $8^{\mathrm{e}}$ classe, les Mittelschulen (groupe hétérogène offrant des passerelles vers les lycées) de la $5^{\mathrm{e}}$ à la $10^{\mathrm{e}}$ classe, et enfin les trois types de lycée (Oberrealschule, Realgymnasium et Gymnasium) allant de la $5^{\mathrm{e}}$ à la $13^{\mathrm{e}}$ classe. Rappelons que seul $1 \%$ d'une classe d'âge obtient l'Abitur en 1921 (pourcentage inchangé en 1930) ${ }^{(5)}$.

L'accent a été mis sur les manuels prussiens, car ce Land se distingue des autres en édictant par décret ministériel du 4 septembre 1920 des consignes précises pour l'enseignement de l'éducation civique, qui doit faire l'objet de cours à part entière en prélevant des heures sur ceux d'histoire et d'allemand. Au niveau du Reich, il n'y a que des recommandations, puisque l'enseignement reste du ressort des Länder après 1918.

Du point de vue de la méthode, c'est une analyse des contenus qui a été choisie. Les manuels ont été étudiés en série, pour évaluer la représentativité de chaque assertion. De grandes tendances ont pu être dégagées: les auteurs du corpus adhèrent majoritairement à la République, tandis que certains sont franchement antirépublicains. Nous avons cherché à mettre en évidence les thèmes consensuels dans la représentation de l'État, même si des oppositions entre auteurs républicains et antirépublicains sont repérables. Au plan sociologique, les auteurs sont quasiment tous enseignants, comme l'indiquent les titres mentionnés en couverture des manuels, le plus souvent dans un établissement secondaire. Plus rares sont ceux qui exercent dans le supérieur (un peu moins de $10 \%$ sont professeurs). Bien qu'ils soient nombreux à être titulaires d'un doctorat (40\% des auteurs), on ne peut toutefois pas les considérer comme des théoriciens. Cette position intermédiaire entre juristes spécialisés en droit constitutionnel et

Baquès, Annie Bruter, Nicole Tutiaux-Guillon (dir.), Pistes didactiques et chemins d'historiens. Textes offerts à Henri Moniot, Paris, L'Harmattan, 2003, p. 161-189; Stephanie Krapoth, «Visions comparées des manuels scolaires en France et en Allemagne ", Cahiers d'histoire, Revue d'histoire critique, 93 (2004), p. 45-62.

3 Emmanuel Droit, L'homme nouveau? L'éducation socialiste en RDA (1949-1989), Rennes, PUR, 2009; Alexandra OEser, Enseigner Hitler. Les adolescents face au passé nazi en Allemagne. Interprétations, appropriations et usages de l'histoire, Paris, Éditions de la MSH, 2010.

4 Voir notamment Alain Choppin, « Manuels scolaires. États et sociétés ", Histoire de l'éducation, $\mathrm{n}^{\circ}$ 58, mai 1993, p. 165-185 et du même auteur, "Le manuel scolaire, une fausse évidence historique ", Histoire de l'éducation, $\mathrm{n}^{\circ} 117$, janvier-mars 2008, p. 7-56.

5 Dieter Langewiesche, Heinz-Elmar Tenorth (éd.), Handbuch der deutschen Bildungsgeschichte, vol. 5 : 1918-1945. Die Weimarer Republik und die NS-Diktatur, Munich, Beck, 1989. 
enseignants ordinaires fait précisément l'intérêt de cette source pour l'histoire culturelle en tant que complément d'une histoire des intellectuels de Weimar ${ }^{(6)}$.

Signalons que le matériau exploité dans cette communication a fait l'objet d'un article paru dans la revue Histoire de l'éducation ${ }^{(7)}$. On a ici privilégié une problématique spécifique centrée sur la notion "d'ordre étatique », qui nous semble centrale dans les manuels (toutes tendances politiques confondues), et on a élargi le corpus aux manuels d'histoire, non pris en compte dans l'article précité. Il convient maintenant d'expliquer comment les auteurs envisagent l'ordre étatique dans le cadre de la république parlementaire nouvellement créée, ce qui pose problème compte tenu de la désaffection dont souffre ce régime depuis 1918.

\section{La valorisation de l'ordre étatique dans les manuels}

\section{L'ordre étatique opposé aux désordres révolutionnaires}

"L’ordre étatique » (Staatsordnung) créé par la Constitution de 1919 est une notion connotée positivement; en effet, on la trouve opposée à la notion (négative) de « désordres » (die Unruhen) engendrés par la défaite et la révolution. Les deux citations suivantes de manuels de 1928 et de 1931 illustrent l'usage fréquent par les auteurs de ce couple antinomique:

«La Constitution de Weimar est devenue le fondement du nouveau Reich. Elle a mis fin aux désordres de la révolution, a restauré le calme et l'ordre. Depuis, le nouveau Reich commence lentement à remonter la pente grâce à un travail obstiné » $(\mathrm{Gehl}, 1928)^{(8)}$.

«Et malgré tout, le peuple allemand est animé par la volonté de fer de se relever. Un important travail, pénible mais payant, a en effet déjà été réalisé ces dernières années: l'État, qui menaçait de se disloquer complètement en 1918, est de nouveau ordonné (Constitution de Weimar, 11 août 1919)» (Kumsteller, 1931) $)^{(9)}$.

La révolution apparaît ainsi, dans la grande majorité des manuels, comme une référence négative, même si ensuite les mêmes auteurs vantent les mérites de la souveraineté populaire, fondement du nouveau régime démocratique. La révolution est d'ailleurs souvent évoquée (plus que véritablement traitée) dans le chapitre intitulé: «Effondrement» («Zusammenbruch»), ce qui illustre cette vision péjorative. De fait, elle est non seulement associée à la défaite et au thème du « coup de poignard dans le

6 Voir notamment Manfred GAngL et Hélène Roussel (dir.), Les intellectuels et l'État sous la République de Weimar, Rennes, Centre de recherches Philia / Paris, Éditions de la MSH, 1993.

7 Marie-Bénédicte Vincent, «'La Constitution doit devenir un livre populaire'. Enseigner le patriotisme constitutionnel sous la République de Weimar », Histoire de l'Éducation, 123, juillet-septembre 2009, p. 71-105.

8 «Die Verfassung von Weimar ist die Grundlage des neuen deutschen Reiches geworden. Sie beendete die Unruhen der Revolution, stellte Ruhe und Ordnung wieder her. Langsam beginnt das deutsche Reich seitdem, durch zähe Arbeit wieder emporzusteigen » (Walter GEHL, Geschichte für höhere Schulen, Gymnasium-Unterstufe, Breslau, Ferdinand Hirt, 1928).

9 «Und trotzdem lebt im deutschen Volk der eiserne Wille, wieder hochzukommen. Viel mühselige, aber erfolgreiche Arbeit ist in der Tat in diesen Jahren bereits geleistet worden: der Staat, der 1918 aus den Fugen zu gehen drohte, ist wieder geordnet (Weimarer Reichsverfassung, 11. August 1919) » (Bernhard Kumsteller, Geschichtsbuch für die deutsche Jugend, Gymnasium-Unterstufe, Leipzig, Verlag Quelle und Meyer, 1931). 
dos » (trahison de l'armée allemande par l'arrière), mais aussi au projet porté par l'extrême gauche non sociale-démocrate d'une dictature du prolétariat. On peut renvoyer à ce sujet à l'étude de Pierre Jardin qui se fonde sur des manuels d'histoire des années 1920 (certains titres de manuels faisant aussi partie de notre corpus) ${ }^{(10)}$.

Par opposition, la Constitution de Weimar est perçue comme un socle mettant fin à ce cycle infernal et ouvrant à terme des perspectives de relèvement pour l'Allemagne, même si souvent les auteurs ne cachent pas leur attentisme. Ainsi dans ce manuel de 1924:

"La Constitution de Weimar crée enfin, émergeant du chaos de l'effondrement, la base - susceptible d'évoluer - d'un nouvel ordre étatique à travers la forme libérale d'une république démocratique fédérale » (Hencke, 1924) ${ }^{(11)}$.

L'une des raisons les plus souvent invoquées par les manuels pour justifier cette dévalorisation de la révolution est que celle-ci a engendré une douloureuse division du peuple allemand, à l'heure où précisément l'union sacrée était le plus nécessaire:

«La révolution n’a pas comblé le fossé qui existe au sein du peuple allemand, mais l'a au contraire accentué. Gauche et droite, républicains et monarchistes, socialistes et capitalistes, internationaux et nationaux, noir-rouge-or et noir-blanc-rouge, Bannière du Reich et groupements patriotiques se font face dans une attitude d'hostilité farouche " $(\mathrm{Gehl}, 1926)^{(12)}$.

À l'inverse, l'ordre constitutionnel de 1919 est valorisé, car il rétablit la paix civile (ou du moins les conditions de celle-ci), autrement dit il ramène l'unité de la nation qui apparaît comme une valeur sacrée pour tous les auteurs, républicains comme antirépublicains. Qu'importe à la limite la forme du régime, ce qu'illustre la citation suivante de 1926 qui ne parle pas de république à propos de la constitution, mais insiste sur les bienfaits d'un ordre intérieur (terme consensuel) au service de l'unité nationale:

«Dans une période extrêmement difficile, le peuple allemand s'est donné une constitution. Elle permet à toutes les composantes du peuple, qui sont naturellement guidées par des conceptions et des désirs différents, de se mettre pacifiquement en valeur au sein de l'État, conformément à leur importance pour le peuple tout entier. C'est ainsi que l'équilibre intérieur est le mieux garanti et par là, la construction de l'unité intérieure dont nous avons besoin par-dessus tout, si notre peuple veut de nouveau occuper dans le monde la position qui lui revient eu égard à son importance économique et culturelle » (Pinnow, 1926) ${ }^{(13)}$.

10 Pierre Jardin, «La légende du 'coup de poignard' dans les manuels scolaires allemands des années 1920 », in: Jean-Jacques Becker, Jay M. Winter, Gerd Krumeich, Annette Becker, Stéphane Audoin-Rouzeau, Guerre et cultures 1914-1918, Paris, Armand Colin, p. 266-277.

11 "Die Weimarer Verfassung schafft schließlich aus dem Chaos des Zusammenbruchs die entwicklungsfähige Grundlage einer neuen Staatsordnung, in der freiheitlichen Form einer bundesstaatlichen demokratischen Republik» (Hencke, Geschichte in Tatsachen. Ein Arbeitsbuch für höhere Anstalten, Heft 2, Breslau, Ferdinand Hirt, 1924).

12 «Die Revolution hat die Kluft im deutschen Volk nicht überbrückt, sondern vertieft: Links und Rechts, Republikaner und Monarchisten, Sozialisten und Kapitalisten, Internationale und Nationale, schwarzrot-gold und schwarz-weiß-rot, Reichsbanner und Vaterländische Verbände stehen sich in erbitterter Feindschaft gegenüber » (Walter GeHL, Geschichte für höhere Schulen, Breslau, Ferdinand Hirt, 1926).

13 «In schwerster Zeit hat sich das deutsche Volk seine Verfassung gegeben. Sie ermöglicht allen Volkskreisen, die naturgemäß von verschiedenartigen Anschauungen und Wünschen geleitet werden, sich innerhalb des Staates in friedlicher Weise, ihrer Bedeutung für das Volksganze entsprechend, zur Geltung zu bringen. So wird am besten der innere Ausgleich gewährleistet und damit die Herausbildung der inneren Einheit, die wir vor allem brauchen, wenn unser Volk wieder die Stellung in der Welt einnehmen 
Le message transmis aux jeunes générations est donc unanime: l'ordre étatique apparaît comme hautement appréciable, car résultant de l'adoption d'une constitution qui, même imparfaite ou considérée comme non définitive, permet tout de même à l'Allemagne de travailler à son relèvement. Ce raisonnement revient à valoriser l'ordre constitutionnel d'août 1919, si ce n'est par un attachement réel (le « cœur») du moins pour des motifs rationnels (la « raison»), pour reprendre les termes employés par l'historien Friedrich Meinecke ${ }^{(14)}$.

\section{La soumission de l'individu à l'ordre étatique}

Dès lors, l'individu a le devoir de respecter cet État et, plus encore, de s'y intégrer. En effet, la représentation de cet État est liée à celle d'une " communauté du peuple ", un terme que les nazis reprendront comme on sait avec succès, mais qui est antérieur: il apparaît entre autres officiellement dans les lignes directrices édictées par le Reich pour l'éducation civique en 1924 (une des missions de l'éducation civique est de consolider la communauté du peuple allemand). Il est intéressant de remarquer ici que les auteurs des manuels lient la notion de communauté du peuple à celle d'ordre constitutionnel (la seconde fournissant le cadre de la première). C'est ce qu'illustrent ces manuels de 1921 et 1930 :

«La plus grande communauté d'hommes est la communauté du peuple. Elle vit dans l'État, s'est donné l'État, comme le prouve l'histoire. Dans la grande communauté du peuple formée par les Allemands, le Reich allemand, il y a des tas de choses différentes à régler. Aucun citoyen ne peut retenir mot à mot toutes les règles. C'est pourquoi on les a mises par écrit et imprimées. L’ordre étatique est appelé Constitution » (Reiniger, 1921) ${ }^{(15)}$.

«C'est pourquoi tu ne t'étonneras pas que dans la Constitution de Weimar on insiste autant sur l'éducation à la communauté du peuple» (Lauterbach, 1930) ${ }^{(16)}$.

Il en découle une relation particulière de l'individu à l'État: l'individu a des « devoirs » envers l'État, en contrepartie des « droits fondamentaux » que lui garantit la constitution. Notons à titre comparatif qu'une "déclaration des devoirs du citoyen " contrebalançant la proclamation des "droits de l'homme et du citoyen " apparaît en France dans la constitution républicaine de l'An III (1795) qui veut mettre fin à la période de désordres ayant suivi la promulgation de la constitution républicaine de l'An I (1793).

soll, die ihm nach seiner wirtschaftlichen und kulturellen Bedeutung zukommt " (Hermann PinNow, Lehrbuch der Geschichte für die Mittelstufe, dritter Teil, Leipzig/Berlin, Verlag von Teubner, 1926).

14 «Tourné vers le passé, je reste monarchiste de cœur, et tourné vers l'avenir, je suis devenu républicain par raison ", cité dans André Gisselbrecht, "Le sort tragique des 'seniors' de Weimar: des républicains par raison », in: GANGL/Roussel (dir.), Les intellectuels et l'État (note 6), p. 25-37.

15 «Die größte Gemeinschaft von Menschen ist die Volksgemeinschaft. Sie lebt im Staat, hat sich den Staat geschaffen (Nachweis aus der Geschichte). In der großen Volksgemeinschaft der Deutschen, im Deutschen Reich, gibt es viel und vielerlei zu regeln. Die einzelnen Regeln kann sich kein Bürger wörtlich merken. Darum hat man sie aufgeschrieben und gedruckt. Die Staatsordnung wird Verfassung genannt» (Max Reiniger, Neue Staatsbürgerkunde, Erster Teil: Die Verfassung in der Schule, 7.-10. Schuljahr, Langensalza, Verlag von Julius Belz, 1921).

16 «Daher nimmst Du nicht wunder, daß in der Verfassung von Weimar so entscheidender Nachdruck auf die Erziehung zur Volksgemeinschaft gelegt wird» (F. LAUter BACH, Unser neues Reich, Geschichte der Deutschen Republik. Zugleich Hilfsbuch für Staatsbürgerkunde, 7.-10. Schuljahr, Halle, Pädagogischer Verlag von H. Schroeden, 1930). 
Ces devoirs sont un appel au respect du droit et de la morale et un éloge de l'ordre et de la propriété (en 1789, les membres du clergé avaient déjà proposé d'assortir à la Déclaration des droits de l'homme et du citoyen une telle déclaration des devoirs, mais ils avaient échoué). Parmi les « devoirs » du citoyen envers la république, on note alors la conscription. Par ailleurs, cette notion de " devoirs du citoyen » est reprise dans la Constitution de la Deuxième République de 1848. C’est dans cette filiation républicaine que s’inscrit la Constitution de Weimar. Mais les devoirs du citoyen sont en Allemagne conçus dans le sens d'une soumission individuelle à l'État pouvant aller jusqu'au « sacrifice » (Opfer). Cette dernière notion est officielle dans les consignes données au niveau du Reich en 1924 pour l'éducation civique, qui doit transmettre aux futurs citoyens le sens des obligations qui leur incombent du fait de leur intégration à l'État et à la communauté du peuple, alors que le service militaire est supprimé par le Traité de Versailles.

"Cela signifie donc servir la communauté. C'est à cela que l'éducation conduit l'homme; quand elle le convainc premièrement de la valeur d'une vie ordonnée de l'État (but de l'éducation civique) et deuxièmement l'habitue au devoir de promouvoir la communauté du peuple et de l'État en s'y intégrant et s'y soumettant volontairement, en tenant compte les uns des autres et, mieux encore, en se sacrifiant volontairement et personnellement " (Reiniger, 1921) ${ }^{(17)}$.

On voit donc qu'avec la notion de sacrifice, les manuels encouragent une attitude affective de l'individu face à l'État, vu comme le garant de l'unité nationale, le lieu d'épanouissement de la communauté du peuple et finalement comme une entité supérieure transcendant l'individu. On est loin d'une construction abstraite faisant de l'État l'addition des volontés des citoyens. Selon cette représentation, l'État n'est pas seulement le lieu de la gestion et de l'administration; il revêt une fonction plus essentielle pour le peuple allemand, puisqu'il réalise son unité. Pour les auteurs, il est clair que cet État ne date pas de l'acte constitutionnel de 1919, mais remonte à la création du Reich en 1871 et perdure par-delà la révolution.

\section{Le thème de la continuité de l'État par-delà la césure de 1918}

Une fois valorisé l'ordre étatique, la grande question à laquelle s'efforcent de répondre directement ou indirectement les auteurs des manuels est celle de l'interprétation de la République de Weimar par rapport au passé allemand: est-elle en rupture ou en continuité avec ce passé? La réponse est cruciale pour évaluer la légitimité de la république, qui peut être perçue soit comme une importation étrangère (ce qui la discrédite dans le contexte de la défaite), soit comme une potentialité de l'histoire allemande et principalement de 1848 . Or, on constate que les manuels renvoient à la représentation d'un État qui préexiste à la césure de 1918, qui évolue dans l'histoire et qui peut donc être modifié dans l'avenir. Cet État transcendant la forme particulière du régime de Weimar est associé à l'appellation de Reich.

17 «Es heißt also: der Gemeinschaft dienen. Dahin führt die Erziehung den Menschen, wenn sie ihn erstens von dem Wert des geordneten Staatslebens überzeugt (Zweck der Staatsbürgerkunde) und ihn zweitens an die Pflicht gewöhnt, unter freiwilliger Einfügung, Unterordnung, gegenseitiger Rücksichtnahme und nicht zuletzt unter freiwilligen persönlichen Opfern die Staats- und Volksgemeinschaft zu fördern » (M. Reiniger, Neue Staatsbürgerkunde [note 15]). 


\section{Un État en devenir}

La plupart des manuels souscrivent à une conception organiciste de l'État inspirée par Aristote et opposée à l'État rousseauiste résultant du contrat social (cette dernière vision étant associée à la France dont l'Allemagne veut se démarquer au plan politique). Par ailleurs, les auteurs mentionnent plusieurs étapes dans l'évolution séculaire de l'État: à l'État féodal a succédé l'État de police, puis l'État de droit et enfin l'État culturel (Kulturstaat), sans qu'une chronologie particulière soit associée à ces étapes. En revanche, les auteurs s'accordent pour caractériser l'État de Weimar comme État culturel, ce qui revient à inscrire le régime dans une continuité historique. Ainsi dans ce manuel de 1921:

« L'État n'est pas né artificiellement, il n'est pas né de traités et d'accords, contrairement à ce que l'on a souvent supposé. On ne peut pas dire qu'il aurait été précédé d'un état sans État. L'État est aussi vieux que l'homme. Au cours des millénaires le concept d'État s'est enrichi à mesure que la culture se développait et se différenciait. L'État a été amené à superviser et prendre à sa charge de plus en plus de branches de l'activité humaine. D’une communauté qui vivait selon des règles frustes et non écrites est né l'État de droit, qui reconnaît le règne de la loi, puis l'État culturel [Kulturstaat], qui se donne pour mission d'encourager toutes les forces économiques et spirituelles de la nation» (Neubauer, 1921) ${ }^{(18)}$.

Il incombe aux auteurs des manuels de souligner à l'attention des élèves ce qui, dans l'ordre étatique de Weimar, relève d'une continuité historique. Montrer cette part de continuité revient à faire accepter le nouvel État (il n'est pas totalement étranger à l'Allemagne, il ne se réduit pas à une importation occidentale, ainsi que le disent ses détracteurs à droite), même si certaines de ces caractéristiques institutionnelles peuvent être contestées. Insister sur la continuité historique, c'est aussi implicitement rappeler le caractère évolutif de cet État, qui peut encore changer (notion de devenir, d'avenir ouvert).

«Les États aussi sont soumis à la loi sacrée du mourir et du devenir. La réorganisation de la vie intérieure de l'État est à considérer de ce point de vue. Il faut établir dans quelle mesure la nouvelle constitution conserve le legs de l'histoire et dans quelle mesure elle a dû faire place à de nouvelles formes et intérêts » (Teubner, 1922) ${ }^{(19)}$.

18 « Nicht künstlich ist der Staat entstanden, nicht durch Vereinbarungen und Verträge, wie man oft angenommen hat. Es ist nicht so, daß ein staatloser Zustand vorangegangen wäre. Der Staat ist so alt wie der Mensch. Im Laufe der Jahrtausende hat sich der Begriff des Staates, je reicher sich die Kultur entwickelte und gliederte, mit einem desto reicheren Inhalt erfüllt. Der Staat hat immer mehr Zweige der menschlichen Tätigkeit in den Bereich seiner regelnden Aufsicht und Fürsorge hineingezogen. Aus einer Gemeinschaft, die nach rohen, ungeschriebenen Bestimmungen lebte, wurde der Rechtsstaat, der die Herrschaft des Gesetzes anerkannte und aus diesem der Kulturstaat, der sich die Förderung aller wirtschaftlichen und geistigen Kräfte der Nation zur Aufgabe stellt » (Friedrich Neubauer, Kleine Staats- und Wirtschaftslehre für höhere Anstalten, Gymnasium-Mittelstufe, Halle, Verlag der Buchhandlung des Waisenhauses, 1921).

19 «Auch die Staaten stehen unter dem heiligen Gesetz des Stirb und Werde. Die Neuregelung des innerstaatlichen Lebens ist unter diesem Gesichtspunkt zu betrachten. Es ist festzustellen, wieweit in der neuen Verfassung historisch Gewordenes erhalten ist, wieweit es neuen Formen und Interessen hat weichen müssen " (Alice SAlomon, Die deutsche Volksgemeinschaft, Wirtschaft, Staat, Soziales Leben. Eine Einführung für Frauenschulen und Fortbildungsschulen, Gymnasium-Oberstufe, Berlin/Leipzig, Verlag Teubner, 1922). 


\section{La continuité du Reich}

La continuité de l'État par-delà le changement de régime s'incarne dans la reprise officielle du terme de Reich dans la Constitution de 1919, un choix assumé dès le départ par les républicains et qui satisfait aux aspirations populaires, comme l'explique ce manuel de 1927:

«Article 1: le nom de l’État est inchangé: Reich allemand. Face aux attaques de l'USPD, le ministre du Reich Hugo Preuß a défendu cette appellation de la manière suivante: le mot Reich a été conservé parce que des traditions séculaires, toute l'aspiration du peuple allemand à l'unité nationale sont attachées au nom de Reich et nous blesserions sans raison des sentiments profondément ancrés si nous voulions renoncer à ce mot» (Treuge, 1927) ${ }^{(20)}$.

Il s'ensuit une dissociation entre l'État-Reich vu comme entité durable et la forme constitutionnelle du régime qui, de monarchique, est devenu républicain, sans que le Reich ne perde son identité. Cette dissociation est théorisée par les juristes spécialistes de droit public, notamment Gerhard Anschütz (1867-1948), qui a rédigé un célèbre commentaire de la Constitution du 11 août 1919 quatorze fois réédité jusqu'en 1933. Certains auteurs de manuels se sont directement servis de ce commentaire, comme l'illustre cet exemple en 1928:

«À la question de savoir comment le Reich allemand actuel se situe par rapport à l'État fédéral de 1871, la majorité des théoriciens du droit public répondent à peu près de cette manière: la révolution allemande de novembre 1918 n’a pas détruit le Reich, mais modifié sa constitution. Le Reich en tant que tel existait et continue d'exister aujourd'hui encore. Les Reich ancien et nouveau valent comme même sujet de droit (Anschütz). C'est aussi ce qu'expriment les premiers mots de la nouvelle constitution du Reich » (Lange, 1928) ${ }^{(21)}$.

Cette représentation d'un Reich dissocié de la forme républicaine du régime a des conséquences extrêmement importantes en termes de culture politique: elle va servir de justification aux antirépublicains dans leur lutte contre le régime de Weimar. Même de hauts fonctionnaires, qui dans leur rhétorique professionnelle continuent de se présenter dans les années 1920 comme des "serviteurs de l'État »(Staatsdiener), vont ouvertement adhérer à des partis de la droite antirépublicaine (DNVP notamment), malgré leur serment de fidélité envers la Constitution de Weimar ${ }^{(22)}$. Notons que les manuels scolaires ne vont pas jusque-là et n'opposent pas frontalement le Reich à la

20 «Artikel 1: 'Der Name des Staates bleibt erhalten: Deutsches Reich'. Gegenüber den Angriffen von seiten der USPD verteidigte der Reichsminister Hugo Preuß diese Benennung mit den Ausführungen: das Wort Reich ist beibehalten worden, weil Traditionen von Jahrhunderten, die ganze Sehnsucht des deutschen Volkes nach nationaler Einigung an dem Namen Reich hängen und wir würden tiefwurzelnde Gefühle ohne Grund und Zweck verletzen, wenn wir von diesem Wort absehen wollten » (Margarete Treuge, Einführung in die Bürgerkunde, Berlin/Leipzig, Teubner, 1927).

21 «Die Frage, in welchem Verhältnis das heutige Deutsche Reich zu dem 1871 geschaffenen Bundesstaat steht, wird von den meisten Staatsrechtslehrern etwa in dem Sinne beantwortet: die deutsche Revolution von November 1918 hat das Reich nicht zerstört, sondern seine Verfassung geändert. Das Reich als solches bestand und besteht auch heute noch weiter. Das alte und das neue Reich gelten als dasselbe Rechtssubjekt (Anschütz). Dies ist auch in den einleitenden Worten der neuen Reichsverfassung zum Ausdruck gebracht » (Karl LANGE, Staatsbürgerkunde für die höheren Schulen des Freisstaates Braunschweig, Gymnasium-Oberstufe, Braunschweig, Kommissionsverlag Rieke, 1928).

22 Voir Marie-Bénédicte Vincent, Serviteurs de l'État, Les élites administratives en Prusse 1871-1933, Paris, Belin, 2006. 
forme républicaine du régime. Mais ils développent néanmoins cette représentation de l'État comme entité transcendant la constitution.

\section{La filiation du régime avec 1848}

Pourtant, les républicains vont s'évertuer à montrer que le régime de Weimar réalise certaines potentialités de l'histoire allemande, principalement celles de 1848, et donc qu'il ne trahit pas le passé, mais qu'il se situe au contraire dans sa continuité. Dans les manuels, le moment 1848 est associé à trois grandes références politiques: les droits fondamentaux, la souveraineté populaire et l'unité nationale. On le voit dans les citations suivantes de 1921 et 1927 :

«Les droits et libertés fondamentaux ont essaimé en Europe depuis la France, ils se trouvent dans les chartes de tous les États constitutionnels. En Allemagne, ces droits fondamentaux apparaissent dans la constitution de l'assemblée nationale de Francfort en 1848 » (Reiniger, $1921)^{(23)}$.

"Le nouveau Reich allemand a réalisé les désirs des hommes [Männer] qui, déjà en 1848, voulaient voir fonder le Reich selon la volonté du peuple. Le Reich allemand est devenu une république » $\left(\right.$ Kolbe, 1927) ${ }^{(24)}$.

Mais l'ambiguïté de la référence historique à 1848 est illustrée par la reprise des couleurs: le drapeau noir-rouge-or fait, pour les auteurs républicains, référence à l'idéal libéral et unitaire, tandis que les auteurs antirépublicains et/ou révisionnistes y voient surtout une allusion à la pensée grande-allemande dans le contexte d'amputation territoriale de l'Allemagne et d'interdiction de l'Anschluß imposées par le Traité de Versailles. Cette double compréhension du drapeau est exprimée par les citations suivantes de 1927 et 1928 :

« La constitution du Reich allemand remonte à l'année 1848. L'assemblée nationale de Francfort réclamait alors un Reich allemand unitaire sous le drapeau noir-rouge-or. La souveraineté du peuple [Volksherrschaft] était sa plus haute revendication. Celle-ci se trouve réalisée dans la nouvelle démocratie [Volksstaat] allemande» (Kolbe, 1927) ${ }^{(25)}$.

«Lors du vote sur les couleurs du Reich, acceptées par une majorité de 190 voix contre 110, le fait que ces couleurs aient valu comme symbole de la pensée nationale grande-allemande incluant l'Autriche pesa lourd dans la balance» (Lange, 1928) ${ }^{(26)}$.

23 «Von Frankreich aus haben diese Freiheits- und Grundrechte ihren Weg durch Europa angetreten, die finden sich in den Verfassungsurkunden aller Verfassungsstaaten. In Deutschland treten diese Grundrechte auch in der Verfassungsurkunde der Frankfurter Nationalversammlung von 1848 auf » (M. ReINIGER, Neue Staatsbürgerkunde [note 15]).

24 «Das neue deutsche Reich hat die Wünsche der Männer zur Verwirklichung gebracht, die schon 1848 das Reich nach dem Volkswillen geschaffen wissen vollten. Das Deutsche Reich ist eine Republik geworden » (H. Kolbe, Eine Einführung in die Entwicklung des deutschen Staats-, Wirtschafts- und Gesellschaftslebens als Grundlage staatsbürgerlicher Bildung und Erziehung für den abschliessenden Geschichtsunterricht, 7.-10. Schuljahr, Langensalza, Verlag von Julius Beltz, 1927).

25 «Die deutsche Reichsverfassung geht zurück auf das Jahr 1848. Die Frankfurter Nationalversammlung verlangte damals ein deutsches Einheitsreich unter der Fahne schwarz-rot-gold. Die Volksherrschaft war die oberste Forderung. Sie ist im neuen deutschen Volksstaat zur Durchführung gekommen » (H. Kolbe, Eine Einführung [note 24]).

26 «Bei der Wahl der Reichsfarben, welche mit einer Mehrheit von 190 Stimmen gegen 110 angenommen wurden, fiel die Tatsache sehr ins Gewicht, daß diese Farben immer als ein Sinnbild des großdeutschen, 
Est ainsi posé le problème des Allemands vivant en dehors des nouvelles frontières $\mathrm{du}$ Reich. Notons ici que tous les manuels se situent plus ou moins dans une perspective révisionniste, certains n'hésitant pas à illustrer à l'aide de cartes géographiques, de statistiques etc. l'étendue des communautés allemandes vivant à l'étranger. Dans les esprits, le Traité de Versailles aggrave la dissociation entre le peuple allemand et l'État allemand. L'État de Weimar n'est pas un État national, il l'est même encore moins que le Kaiserreich.

"La paix imposée de Versailles ne nous a fait que trop prendre conscience de ce que l'appartenance au peuple et l'appartenance à l'État ne se recouvrent pas toujours, loin de là. Une partie du sol allemand, habitée par des Allemands, a été arrachée à notre patrie sur décision autoritaire et rattachée à des États étrangers" (Niebuhr, 1928) ${ }^{(27)}$.

$\mathrm{Au}$ total, on voit que le thème de la continuité de l'État est ambivalent: pour les républicains, il s'agit de montrer que la République de Weimar réalise complètement les idéaux unitaire et démocratique de 1848, ce qui légitime le régime. Les antirépublicains insistent, eux, sur la continuité du Reich (celui de 1871), qui perdure malgré la révolution de 1918/19 et qui constitue de la sorte une réalité supérieure à la forme républicaine du nouveau régime. Cette dernière représentation ouvre la voie à une possible contestation de la république. En effet, celle-ci apporte un certain nombre de nouveautés que les auteurs ne peuvent nier.

\section{Les nouveautés apportées par l'ordre étatique de Weimar et leurs limites}

\section{L'État unitaire du peuple}

Très pédagogiquement, un bon nombre de manuels vont expliquer en quoi la Constitution de Weimar diffère de celle du Reich de 1871. Les deux principales innovations sont d'une part le centralisme et d'autre part le caractère démocratique du régime. Or, si le Reich de 1871 est créé comme État fédéral, un processus de centralisation a vu le jour dès les années 1870 et 1880 dans l'organisation de la justice, le système de poids et de mesures ou la législation sociale. La Première Guerre mondiale a accéléré ce processus avec la mise en place de l'économie dirigée; parallèlement, la guerre a entraîné une nationalisation de la population qui, pour certaines de ses composantes, parfois pour la première fois, a identifié le Reich à une "patrie " (Vaterland). Si bien que lors de la création de la République de Weimar, l'évolution vers un État unitaire semble aller de soi. Dans le nouveau Reich, les anciens États sont devenus des Länder qui, sans perdre toute leur souveraineté, doivent désormais s'incliner face au Reich: «Le droit du Reich casse le droit du Land» (article 13 de la Constitution de 1919). Les auteurs des manuels ne contestent pas cette évolution, même s'ils se partagent en deux grandes tendances: ceux qui, par enthousiasme, accentuent le caractère unitaire du

Österreich in die nationale Einheit einschließenden Gedankens gegolten hatten » (K. LANGE, Staatsbürgerkunde [note 21]).

27 «Der Gewaltfrieden von Versailles hat uns so recht zum Bewußtsein gebracht, daß sich Volks- und Staatsangehörigkeit längst nicht immer decken. Deutscher Volksboden, von Deutschen bewohnt, wurde durch Machtspruch von unserem Vaterland losgerissen und fremden Staaten angegliedert » (Albrecht Niebuhr, Wichtige Wissensstoffe für die Landjugend. Sachlese und Lernbuch für die ländliche Fortbildungsschulen, 3. Teil, Lüneburg, Verlag von Herold und Mahlstab, 1928). 
Reich (citation suivante) et ceux qui insistent au contraire sur le maintien des Länder et sur l'héritage du fédéralisme jugé typiquement allemand (notamment les auteurs non prussiens).

«La république allemande n'est donc pas un État fédéral [Bundesstaat] comme l'était le Reich de 1871-1918. Dans la constitution du Reich on ne parle plus d'États fédérés ou d'États membres, mais de Länder. Le nouveau Reich est un État unitaire du peuple [einheitlicher Volksstaat], fondé par l'ensemble de la nation allemande »(Reiniger, 1921) ${ }^{(28)}$.

Un manuel d'éducation civique de 1927 destiné aux Mittelschulen va jusqu'à expliquer que la centralisation apportée par la Constitution de Weimar parachève l'œuvre unificatrice de Bismarck, ce qui revient à relativiser la césure de 1919 et à la faire accepter.

«Déjà Bismarck avait souhaité un Reich unitaire. S'il n’a pu fonder qu’un État fédéral, c'est qu'il lui était impossible de briser les résistances d'alors. Comme il le disait lui-même, Bismarck a dissimulé le caractère étatique du Reich pour gagner les princes à la cause unitaire» (Kolbe, 1927) ${ }^{(29)}$.

La véritable rupture introduite par la Constitution de 1919 concerne, pour les auteurs, la souveraineté populaire vue comme le fondement de la démocratie. C'est le critère fondamental permettant d'opposer la République de Weimar au Kaiserreich. Certains manuels font une analyse comparée des préambules des constitutions de 1871 et 1919. Cette rupture est assumée:

"La première différence fondamentale avec la constitution du Reich de 1871 réside dans la disposition selon laquelle le pouvoir étatique émane du peuple. Cela est exprimé dès l'introduction: "Le peuple allemand, uni dans ses lignées et animé de la volonté de rénover et d'affermir son Reich dans la liberté et la justice, de servir la paix intérieure et extérieure et de promouvoir le progrès social, s'est donné cette constitution” " (Pinnow, 1930) ${ }^{(30)}$.

\section{La méfiance vis-à-vis de la démocratie parlementaire}

Toutefois, il subsiste chez les auteurs une méfiance vis-à-vis de la démocratie. Le terme lui-même est très rarement employé, sans doute à cause de sa consonance occidentale (c'est moins vrai pour l'adjectif demokratisch). L'État de Weimar est qualifié « d'État du peuple " (Volksstaat), ce qui pour les auteurs rend compte de son caractère démocratique. Mais certains (minoritaires il est vrai) disent explicitement que la souveraineté du

28 «Die deutsche Republik ist also kein Bundesstaat wie das Reich von 1871-1918 einer war. In der Reichsverfassung wird nie mehr von Einzelstaaten oder Gliedstaaten sondern von Ländern gesprochen. Das neue Reich ist ein einheitlicher Volksstaat, den die gesamte deutsche Nation begründet hat » (M. ReINIGER, Neue Staatsbürgerkunde [note 15]).

29 «Schon Bismarck hatte nach dem Einheitsreich gestrebt. Er konnte nur den Bundesstaat schaffen, weil es ihm nicht möglich war, die damaligen Widerstände zu brechen. Bismarck hatte, wie er selbst sagte, die Staatlichkeit des Reichs verschleiert, um die Fürsten für das Einheitswerk zu gewinnen » (H. Kolbe, Eine Einführung [note 24]).

30 « Der erste grundlegende Unterschied zur Reichsverfassung von 1871 liegt in der Bestimmung, daß die Staatsgewalt vom Volk ausgeht. Das kommt schon in der Einleitung zum Ausdruck: 'Das deutsche Volk, einig in seinen Stämmen und von dem Willen beseelt, sein Reich in Freiheit und Gerechtigkeit zu erneuern und zu festigen, dem inneren und äußeren Frieden zu dienen und den gesellschaftlichen Fortschritt zu fördern, hat sich diese Verfassung gegeben' " (Pinnow, Kappert und Berger, Deutsche Geschichte in Längsschnitten, Gymnasium-Mittelstufe, Leipzig/Berlin, Teubner, 1930). 
peuple peut s'incarner dans un régime non républicain, par exemple dans une monarchie parlementaire sur le modèle britannique. Le régime monarchique en tant que tel n'est d'ailleurs pas condamné dans les manuels qui se bornent à présenter de manière neutre une typologie des régimes politiques existant dans le monde. Les manuels de Weimar s'opposent ici nettement aux manuels français de la Troisième République qui condamnent le régime monarchique, notamment le régime bonapartiste. La méfiance vis-à-vis de la démocratie vue comme une importation occidentale est explicite dans le manuel écrit en 1927 par une des rares femmes présentes comme auteurs dans le corpus étudié:

« Il revient à la jeune république, le Reich allemand, de se doter, si l'État veut s’avérer viable, de son propre aménagement intérieur, indépendamment de l'étranger. Certes nous voulons apprendre de l'étranger, mais pas l'imiter, car la démocratie ne pourra être conservée chez nous que si elle devient une démocratie allemande» (Treuge, 1927) ${ }^{(31)}$.

De même, la défiance envers le parlementarisme est assez communément répandue dans les manuels. "Le parlementarisme est calqué sur les puissances occidentales » écrit Gehl dans son manuel d'histoire de $1926^{(32)}$. Les auteurs reprochent au parlementarisme de porter en germe la division des Allemands et se méfient du pouvoir excessif dont dispose le Reichstag à leurs yeux. Tous les manuels s'accordent d'ailleurs sur la critique des partis politiques qui, selon eux, poursuivent des intérêts égoïstes et partisans, loin de l'intérêt général: ils menacent ainsi la cohésion de la communauté du peuple que réclame pourtant la situation de l'Allemagne depuis la défaite.

«L'exercice du droit de vote divise notre peuple en une multitude de partis, qui jusqu'à présent, même dans le nouveau Reich, n’ont pas réussi à mettre au premier plan les points de vue communs à tous les Allemands ni donc à engager l'unification si nécessaire, mais s'opposent toujours de manière haineuse. Pour que notre peuple puisse à nouveau se relever de ce profond déclin, il est urgent que nous trouvions, par-delà les conceptions qui divisent, ce qui nous est commun à tous " (Otto, 1921) ${ }^{(33)}$.

Par opposition au Parlement, le Reichspräsident apparaît comme le garant de l'unité intérieure. Les auteurs le décrivent comme situé au-dessus des querelles partisanes, protecteur de l'ordre étatique (valeur consensuelle comme on l'a vu). On retrouve ici en filigrane la dissociation entre une entité étatique respectée de tous et la forme républicaine du régime, sujette elle à contestation. Le président du Reich est même comparé dans plusieurs manuels à l'ancien empereur, du fait de prérogatives similaires.

31 «Der jüngsten Republik, dem Deutschen Reich, kommt es zu, sich unabhängig vom Ausland die eigene Inneneinrichtung zu schaffen, wenn sich der Staat als lebenskräftig erweisen soll. Wohl wollen wir vom Ausland lernen, es aber nicht nachahmen, denn die Demokratie wird bei uns nur zu erhalten sein, wenn sie sich zur deutschen Demokratie entwickelt » (M. Treuge, Einführung in die Bürgerkunde [note 20]).

32 «Der Parlamentarismus ist den westeuropäischen Mächten nachgebildet » (W. GEHL, Geschichte für höhere Schulen [note 12]).

33 «Die Ausübung des Wahlrechts zersplittert unser Volk in einer Anzahl von Parteien, die es bisher auch im neuen Reich nicht verstanden haben, die allen Deutschen gemeinsamen Gesichtspunkte in den Vordergrund zu rücken und dadurch die so notwendige Einigung anzubahnen, sondern die sich immer feindselig gegenüberstehen. Soll unser Volk wieder emporsteigen aus dem tiefen Niedergange, so ist es dringend notwendig, daß wir über den trennenden Auffassungen das herausfinden, was uns allen gemeinsam ist » (Н. Отто, Bürgerkunde für Schule und Haus, 7.-10. Schuljahr, Berlin, Verlag Paul Parey für Landwirtschaft, Gartenbau und Forstwesen, 1921). 
«Le président du Reich est institué en gardien de l'État. Il doit préserver l'État, quand par exemple certains partis du Parlement placent leurs intérêts particuliers au-dessus du bien commun. Le président du Reich doit se situer au-dessus des partis » (Kolbe, 1927) ${ }^{(34)}$.

«Le président du Reich est un organe de la république allemande doté de pouvoirs extraordinaires. Sa position ressemble à plusieurs égards à celle de l'ancien empereur allemand » (Otto, 1921) $)^{(35)}$.

On lit ici en filigrane la préférence des auteurs pour un régime de type présidentiel, une potentialité contenue comme on le sait dans la Constitution de Weimar à l'article 48, qui autorise le président du Reich à gouverner par décrets sans le Parlement en cas de circonstances exceptionnelles (ce qui se produisit effectivement à partir de 1930).

\section{Les restrictions apportées par le Traité de Versailles}

Par ailleurs, le nouvel ordre étatique est soumis à des contraintes imposées par le Traité de Versailles. Les manuels d'histoire comme d'éducation civique consacrent parfois un chapitre entier au Diktat, certains vont jusqu'à le citer in extenso en annexe. Sa dénonciation en tant que telle s'accompagne d'un commentaire sur la manière dont il pèse sur la Constitution.

«Ainsi, la république allemande est, par sa forme, la démocratie la plus démocratique du monde. En vérité elle est pourtant limitée de manière extrêmement sensible au plan politique et économique par les dispositions de la paix de Versailles » (Jaenicke, 1923) ${ }^{(36)}$.

«L'article 178 porte atteinte à la souveraineté et à la constitution du Reich, car il dit que les dispositions constitutionnelles sont suspendues si elles ne sont pas conformes au traité de paix de Versailles. Ainsi, tout le tragique de l'histoire allemande se trouve encore une fois condensé dans cette disposition » (Schnabel, 1928) ${ }^{(37)}$.

Cette dernière citation mérite d'autant plus l'intérêt qu'elle émane de Franz Schnabel (1887-1966), professeur d'histoire de 1922 à 1936 à l'Université technique de Karlsruhe, connu pour être l'auteur en 1929 d'un ouvrage célèbre, Deutsche Geschichte im 19. Jahrhundert, plusieurs fois réédité après 1945. Or Schnabel vouait un attachement sans faille à la démocratie parlementaire de Weimar (ce qui lui coûta son poste en 1936).

Comme on le sait, la concomitance néfaste entre l'instauration de la première démocratie en Allemagne et le traumatisme de la défaite militaire a hypothéqué le

34 «Der Reichspräsident ist zum Hüter des Staates gesetzt. Er soll den Staat bewahren, wenn etwa einzelne Parteien im Reichstag ihre besonderen Interessen über das Wohl der Gesamtheit stellen. Der Reichspräsident soll über den Parteien stehen » (H. KolbE, Eine Einführung [note 24]).

35 «Der Reichspräsident ist ein mit außerordentlichen Machtmitteln ausgestattes Organ der deutschen Republik. Seine Stellung ähnelt in vieler Beziehung der des früheren deutschen Kaisers » (Н. Отто, Bürgerkunde für Schule und Haus [note 33]).

36 "Somit ist der Form nach die Deutsche Republik die 'demokratischste Demokratie' der Welt, in Wahrheit wird sie jedoch durch die Bestimmungen des Versailler Friedens politisch und wirtschaftlich auf das empfindlichste eingeschränkt » (JAENICKE, Staatsbürgerkunde für höhere Anstalten und Fachschulen, Berlin, Car Heymann, Gymnasium-Mittelstufe, 1923).

37 «Denn Souveränität und Verfassung des Reiches sind angestastet durch Artikel 178, welcher erklärt, daß Verfassungsbestimmungen ruhen, wenn sie nicht übereinstimmen mit dem Friedensvertrag von Versailles. So ist in dieser Bestimmung die ganze Tragik der deutschen Geschichte noch einmal zusammengedrängt » (Franz Schnabel, Grundriß der Geschichte für die Oberstufe, GymnasiumOberstufe, Berlin/Leipzig, Teubner, 1928). 
développement serein de la République de Weimar. Mais les historiens récusent aujourd'hui tout déterminisme dans l'histoire de cette république. Les circonstances difficiles de sa naissance l'ont certes fragilisée, mais sans rendre sa fin nécessaire: une autre issue était possible ${ }^{(38)}$.

\section{Conclusion}

La notion «d'ordre étatique » est donc centrale dans les manuels d'histoire et d'éducation civique de la République de Weimar. Elle touche à l'interprétation du nouveau régime et à la manière de le situer par rapport au passé allemand. Aux désordres révolutionnaires est opposé un ordre constitutionnel nécessaire au relèvement de l'Allemagne. Cet ordre constitutionnel présente certes des nouveautés par rapport au Kaiserreich (centralisme relatif, souveraineté du peuple), mais sans être en rupture totale avec le passé allemand. C'est précisément ce thème de la continuité de l'État par-delà la césure de 1918/19 qui apparaît comme une idée-force des auteurs, républicains comme antirépublicains. Les premiers peuvent ainsi légitimer la République de Weimar comme réalisant des potentialités de l'histoire allemande (principalement les idéaux de 1848), tandis que les seconds s'appuient sur la continuité du Reich pour élaborer la représentation d'un État transcendant la forme particulière des régimes, ce qui leur permet de relativiser l'importance de la démocratie pour le pays.

Par ailleurs, les auteurs des manuels expliquent souvent dans leur préface ou leur conclusion que leur but est d'éveiller chez les élèves un lien affectif avec l'État. Ce qui compte pour eux, c'est de susciter un « attachement à l'État » (Staatsgefühl), et non un attachement à la démocratie. Notons que dans la Constitution de la Deuxième République en France (4 novembre 1848), le préambule affirme que « les citoyens doivent aimer la patrie, servir la république, la défendre au prix de leur vie ». Et dans les manuels d'histoire de la Troisième République, les auteurs s'emploient à diffuser un amour de la république ou de la patrie ${ }^{(39)}$, mais pas de l'État. On voit donc ici une différence entre les cas allemand et français dans la hiérarchie des valeurs (l'État primant en Allemagne sur la république).

Reste bien sûr à savoir, et c'est une limite de cette étude, quelle est la réception de cette littérature scolaire en Allemagne, tant chez les enseignants (nombre d'heures de cours effectivement consacrées à l'éducation civique dans les emplois du temps et part de résistance face aux consignes émanant du Reich) que chez les élèves. Les sources ici étudiées ne permettent pas de répondre à ces questions pourtant importantes de l'histoire culturelle.

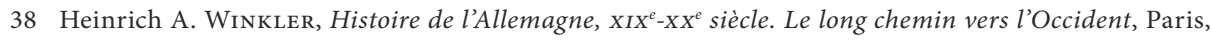
Fayard, 2005 pour la trad. française.

39 Patrick Garcia et Jean Leduc, L'enseignement de l'histoire en France de l'Ancien régime à nos jours, Paris, Armand Colin, 2003; Evelyne Hery, Un siècle de leçons d'histoire. L'histoire enseignée au lycée 1870-1970, Rennes, PUR, 1999. 
Annexe: Corpus des manuels scolaires d'histoire et d'éducation civique de la République de Weimar consulté au Georg-Eckert-Institut für internationale Schulbuchforschung de Brunswick

\section{Les manuels d'histoire}

Unterstufe (cote HCG III)

Prof. Dr. Franz Bender (Studienrat am Gymnasium der Stadt Köln in der Kreuzgasse), Geschichtsbilder für die deutsche Jugend, Francfort-sur-le-Main, Verlag Moritz Diesterweg, 1928 (2 éd. 1931), 96 p.

Walther GeHL, Geschichte für höhere Schulen, Unterstufe: Lebensbilder aus der deutschen Geschichte, mit 59 Bildern im Text und einer Bildtafel, Breslau, Ferdinand Hirt, 1928, 104 p.

Prof. Dr. Bruno Gumlich (Studiendirektor in Charlottenburg) und Dr. Wilhelm TAube (Oberstudienrat in Lichterfelde bei Berlin), Bilder aus der deutschen Geschichte, Vorstufe zum deutschen Lehrbuch der Geschichte "Völkerschicksale », Francfort-sur-le-Main, Verlag Moritz Diesterweg, 1927 (2 éd.), 91 p.

Hencke (Studienrat an der Riehlschule in Biebrich a. Rhein), Geschichte in Tatsachen, Vorstu$\mathrm{fe}$, Deutsche Geschichte von der ältesten bis auf die jüngste Zeit mit 18 Abbildungen, Breslau, Ferdinand Hirt, 1925, 46 p.

Kaemmel, Ulbricht, Sснmidt, Aus Geschichte und Sage, Geschichtliches Unterrichtswerk für sächsische höhere Lehranstalten (Vorstufe, Sexta und Quinta), Meißen, Verlag Schlimpert \& Püschel, 1926, 149 p.

Dr. Bernhard Kumsteller, Geschichtsbuch für die deutsche Jugend, für die Kleinen (Unterstufe), Leipzig, Verlag von Quelle und Meyer, 1931, 88 p.

Friedrich Neubauer (geh. Studienrat), Grundzüge der Geschichte für höhere Lehranstalten, 1. Teil, 2 Bändchen: Deutsche Geschichte seit Anfang des 18. Jahrhunderts für die Unterstufe, Halle, Buchhandlung des Waisenhauses, 1924, $72 \mathrm{p}$.

Arnold Reimann (Direktor des Berliner Gymnasiums zum Grauen Kloster), Das Heldenbuch, Erzählungen aus der deutschen Geschichte für den Anfangsunterricht, 1. Teil: Unterstufe, Munich/Berlin, Verlag Oldenbourg, 1926, 150 p.

Dr. Rudolf Schulze (Studienrat am Paulinischen Gymnasium Münster), Mein Deutschland! Geschichtsbilder aus dem Werdegang des deutschen Volkes, für die Quinta höherer Lehranstalten, mit 36 Bildern, Paderborn, Schöningh Verlag, 1925, 114 p.

Teubners Geschichtliches Unterrichtswerk für höhere Lehranstalten, 1. Bd.: Geschichtserzählungen für die Unterstufe (in Verbindung mit Dr. H. Gräfin von Pestalozza in Berlin, dargestellt von Th. Steudel), Leipzig/Berlin, Verlag Teubner, 1926, 94 p.

\section{Mittelstufe (cote HDG III)}

Prof. Dr. F. zu Bonsen in Münster, Lehrbuch der Geschichte für Lyzeen, Düsseldorf, Verlag von Schwann, 1924, $382 \mathrm{p}$.

Dr. J. Brüggemann, Dr. F. Leonard und Dr. R. Schulze, Steins Lehrbuch der Geschichte, für höhere Lehranstalten, Mittelklassen, 3. Teil: Die Neuzeit (von 1648 bis zur Gegenwart), Paderborn, Ferdinand Schöningh, 1931 (26 éd.), 188 p.

Friedrich Dorner und Kurt Hoffmann, Deutsches Werden, Geschichtsunterricht für die höheren Unterrichtsanstalten, 4. Bd.: Neueste Zeit, Bamberg, Buchners Verlag, 1928, 184 p.

Dr. Walter GeHL, Geschichte für höhere Schulen, Mittelstufe: ein Hilfsbuch zu geschichtlicher Anschauung, 4. Heft: Neueste Zeit (1815-1925), Breslau, Ferdinand Hirt, 1926 (3éd.), 79 p.

Dr. Groebe, Kochs Lehrbuch der Geschichte für höhere Lehranstalten, Mittelstufe, Teil II: Geschichte des deutschen Volkes von der französischen Revolution bis zur Gegenwart, Leipzig, Verlag Quelle und Meyer, 1926 (6éd.), 117 p.

A. Hencke (Studienrat an die Riehlschule in Biebrich am Rhein), Geschichte in Tatsachen, ein Arbeitsbuch für höhere Lehranstalten, Heft 2: Neuere Geschichte seit der französischen 
Revolution, Neueste Geschichte, Leitlinien zur Staatsbürgerkunde mit 17 Karten, Breslau, Ferdinand Hirt, 1924, 43 p.

Dr. Joseph Habisreutinger (Studienrat an der Gisela Oberrealschule in München), Ebner, Geschichte der Neuzeit, Bamberg, Buchners Verlag, 1932 (24 et $25^{\mathrm{e}}$ éd.), 220 p.

Dr. Ludwig Humborg (Studienrat am städtischen Gymnasium zu Münster), Welters Lehrbuch der Weltgeschichte, 4. Teil: Neueste Zeit, die Zeit des Imperialismus, 40. Auflage mit 198 Quellenauszügen, 69 Karten, 24 Diagrammen, 10 Bildnissen und Zeittafel, Münster, Universitätsbuchhandlung Franz Coppenrath, 1928, 271 p.

Dr. Bernhard Kumsteller in Verbindung mit Dr. Ulrich HaAcke und Dr. Benno Schneider, Geschichtsbuch für die deutsche Jugend, Mittelstufe, Leipzig, Verlag von Quelle und Meyer, 1926 (20 éd.), 293 p.

Dr. Alfred Maurer (Studiendirektor), Maier-Schirmeyer Lehrbuch der Geschichte, Mittelstufe, 4. Bd. : Von 1815 bis zur Gegenwart, Francfort-sur-le-Main, Verlag Moritz Diesterweg, 1928 (3éd.), 144 p.

Adolf Meyer (Studienrat in Altona), Die neueste Zeit von 1815 bis zur Gegenwart für die Mittelstufe, 5. Band mit 78 Abbildungen und 42 Tabellen in Anhang (Geschichtswerk für höhere Schulen), Munich/Berlin, Verlag Oldenbourg, 1932, 227 p.

Dr. Friedrich Neubauer, Grundzüge der Geschichte für höhere Lehranstalten, 5. Teil: Vom Wiener Kongreß bis zur Gegenwart, für die Mittelstufe, Halle, Buchhandlung des Waisenhauses, $1923,103 \mathrm{p}$.

A. Pfennings (Oberschulrätin) und M. Stolze (Studienrätin), Im Wandel der Zeiten. Ein Geschichtsbuch für deutsche Mädchen, Mittelstufe, 4. Teil: Von 1815 bis zur Gegenwart, Untersekunda, bearbeitet von M. Nürnberg und C. Müller, Paderborn, Ferdinand Schöningh, 1932, 204 p.

Dr. H. Pinnow (Oberstudienrat in Kassel), in Verbindung mit Th. STeudel (Studienrat in Erfurt) und Dr. E. Wilmanns (Oberstudiendirektor in Barmen), Geschichtsbuch für die Mittelstufe (Teubners Geschichtliches Unterrichtswerk für höhere Lehranstalten), 4. Teil mit 33 Abbildungen, Leipzig/Berlin, Verlag von Teubner, 1926, 234 p.

Dr. Hermann Pinnow (Oberstudienrat in Kassel), Lehrbuch der Geschichte für die Mittelstufe, dritter Teil: Geschichte des deutschen Volkes von 1648 bis zur Gegenwart, Leipzig/Berlin, Verlag von Teubner, 1926, $163 \mathrm{p}$.

Dr. Hermann Pinnow (Oberstudienrat in Kassel), Kappert (Studiendirektor in Hohenstein in Ostpreußen) und BERGER (Mittelschullehrerin in Krefeld), Deutsche Geschichte in Längsschnitten, Teubners Geschichtliches Unterrichtswerk für höhere Anstalten, Leipzig/Berlin, Teubner, 1930 (2 éd.), 167 p.

Dr. Friedrich VogeL, Lehrbuch für den ersten Unterricht in der Geschichte, 3. Bd. : Deutsche Geschichte der Neuzeit mit besonderer Berücksichtigung der bayerischen Geschichte, Bamberg, Buchners Verlag, 1931 (10éd.), 147 p.

Oberstudiendirektor Dr. F. Wuessing und Studienrat Dr. G. Wenz, Aus deutscher Geschichte, 3. Teil: Deutscher Hort: kulturkundliches Lesebuch für die höheren Schulen in zehn Heften, Heft 18, 10. Schuljahr, Leipzig, Quelle und Meyer, 1925, 48 p.

\section{Oberstufe (cote HEG)}

Dr. Franz Schnabel (Prof. an der technischen Hochschule in Karlsruhe), Grundriß der Geschichte für die Oberstufe, Teubners geschichtliches Unterrichtswerk für höhere Anstalten, Teil 4: Geschichte der neuesten Zeit, Ausgabe C, Berlin/Leipzig, Teubner, 1928, 180 p.

Dr. Walther GeHL, Geschichte für höhere schulen, Oberstufe, Ein Hilfsbuch zu geschichtlichem Denken und Dehnen, 4. Heft: Neueste Zeit, Breslau, Ferdinand Hirt, 1925, 159 p.

\section{Les manuels d'éducation civique}

7-8 Schuljahr, Volksschule (cote SDH)

Walther HARDT (Rektor), Präparationen für den Geschichtsunterricht in Volksschulen, mit besonderer Berücksichtigung der ministeriellen Richtlinien zur Aufsstellung von Lehrplänen für 
die oberen Jahrgänge der Volksschulen, 3. Bd.: Staatsbürgerkunde, Leipzig, Richard Danehl's Verlag, 1925 ( $3^{e}$ éd.), 340 p.

Peter Herz, Bürgerkunde. Merkbuch für Schüler an Volks- und Berufsfortbildungsschulen, Kempten, Verlag Bürgerkunde Kempten, 1927 (7éd.), 48 p.

Otto JANKe (Rektor in Berlin), Aus der Staats- und Bürgerkunde und dem Wirtschaftsleben. Zeitgemäßes für Volks- und Fortbildungsschule, Habelschwerdt, Verlag der Deutschen Lehrerbücherei, 1920, $63 \mathrm{p}$.

J. Рӧтsсн (Rektor), Die Reichsverfassung in der Volksschule, Präparationen für eine Oberklasse, Paderborn, Verlag Ferdinand Schöningh, 1921, 40 p.

Otto SeELING (Rektor in Berlin), Staatsbürgerkunde für Volksschulen, Leipzig, Verlag von Max Gehlen, 1921, 48 p.

Margarete Treuge, Einführung in die Bürgerkunde, Berlin/Leipzig, Teubner, 1927 (6 éd.), 280 p.

\section{7-10 Schuljahr (cote SD)}

Rektor A. BoDEsoHn, Leitfaden der Staats- und Bürgerkunde, Ein Lehr-ud Arbeitsbuch für die Hand der Schüler in Fortbildungsschulen, Mittelschulen und in der Oberstufe der Volksschulen, Wittenberg, R. Herrosé's Verlag, 1921 ( $2^{\mathrm{e} e ́ d}$.), 132 p.

A. Bodesohn, Handbuch der Staats- und Bürgerkunde. Ein Lehr- und Lesebuch für den Unterricht in Schulen sowie zum Selbstunterricht, Wittenberg, Herrosé, 1921 (3e éd.), 1923 (4éd.), 1927 (6 éd.), 455 p.

Ulrich HaAcke und Benno Schneider, Dein Staat und Dein Volk, Eine Staatsbürgerkunde, Leipzig, Verlag von Quelle und Meyer, 1928 ( $1^{\text {re }}$ éd.), 1930 (4 éd.), 124 p.

Karl Номвurg (Seminarlehrer), Staatsbürgerkunde. Ein Hilfsbuch für den gemeinschaftskundlichen Unterricht sowie für methodische Unterweisungen an Lehrerbildungsanstalten, Osterwieck-Harz/Leipzig, Verlag von A. W. Zickfeldt, 1920, 1923 (4 éd.), 86 p.

H. Kolbe, Eine Einführung in die Entwicklung des deutschen Staats-, Wirtschafts- und Gesellschaftslebens als Grundlage staatsbürgerlicher Bildung und Erziehung, für den abschliessenden Geschichtsunterricht, Langensalza, Verlag von Julius Beltz, 1927, 119 p.

F. Lauterbach, Unser neues Reich. Geschichte der Deutschen Republik. Zugleich Hilfsbuch für Staatsbürgerkunde, Halle, Pädagogischer Verlag von Hermann Schroedel, 1930, 128 p.

Dr. Georg Friedrich Muth, Lerngerüst zur Kunde von der Gemeinschaft, Staatsbürgerkunde auf freigesamtunterrichtlicher Grundlage, Verlag Dernbruck Neu-Isenburg, 1931, 28 p.

H. Отто, Bürgerkunde für Schule und Haus, Berlin, Verlag Paul Parey für Landwirtschaft, Gartenbau und Forstwesen, 1921 ( $3^{e}$ éd.), 183 p.

Max Reiniger (Kreisschulrat in Bonn), Neue Staatsbürgerkunde, 1. Teil: Die Verfassung in der

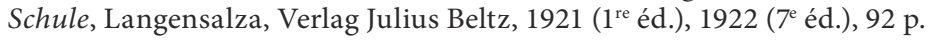

Max ReIniger, Neue Staatsbürgerkunde, Zweiter Teil: Reichsgesetzgebung, Rechtspflege, Deutsche Verfassungsgeschichte, Der Friedensvertrag von Versailles, Verkehrswesen, Geschichte des Handwerks, die soziale Entwicklung, Geschichte der Landwirtschaft und des Bauernstandes, Geschichte des deutschen Hauses, Langensalza, Julius Beltz, 1921, 1922 ( $2^{e}$ éd.), 1923 (3 éd.), 1925 ( $4^{\mathrm{e}}$ éd.), 1927 (5éd.), 1929 (6éd.), 148 p.

Paul Wels (Studienrat an der öffentlichen Handelslehranstalt und Wirtschaftsoberschule in Dresden), Staatsbürgerkunde für Sachsen. Für Schulen, Unterrichtskurse und zum Selbstunterricht, Dresde, Ehlermann, 1927 ( $4^{\mathrm{e}}$ éd.), 50 p.

\section{Mittelstufe Gymnasium (cote SDG)}

Dr. Hermann JAENICKE, Staatsbürgerkunde, für höhere Lehranstalten und Fachschulen sowie zum Selbstunterricht, Berlin, Car Heymanns Verlag, 1923, 1929 (2 éd.), 140 p.

Dr. Joseph Kösters (Studiendirektor), Deutsche Staatsbürgerkunde für höhere Schulen, Münster, Verlag von Heinrich Schöningh, 1924, 48 p.

Friedrich Neubauer, Kleine Staats- und Wirtschaftslehre für höhere Lehranstalten, Halle, Verlag der Buchhandlung des Waisenhauses, 1921 (5éd.), 1926 (6éd.), 103 p. 


\section{Oberstufe, Gymnasium (cote SEG-III)}

Prof. Dr. Phil. Hans Kania (Studienrat am staatlichen Viktoria-Gymnasium in Potsdam), Staatsbürgerkunde auf Grund vergleichender geschichtlicher Übersichten, Leipzig/Berlin, Teubner Verlag, 1921 ( $2^{\mathrm{e}}$ éd.), 1922 (3éd.), 1926 (4éd.), 1928 (5éd.), 80 p.

Dr. Karl Lange (Studienrat an der Raabeschule, staatliche Realschule zu Braunschweig), Staatsbürgerkunde für die höheren Schulen des Freisstaates Braunschweig, Braunschweig, Kommissionsverlag Rieke, 1928, 1930 ( $2^{\mathrm{e}}$ éd.), 128 p.

Elisabeth Mleinek, Staatsbürgerkunde in der Mädchenbildung, Paderborn, Verlag Ferdinand Schöningh (coll. Mädchenbildung in der Volksschule, Schriftenreihe des Vereins katholischer deutscher Lehrerinnen, herausgegeben vom Volksschulausschuß, Bd. 5), 1930, 159 p.

Alice Salomon, Die deutsche Volksgemeinschaft, Wirtschaft, Staat, Soziales Leben. Eine Einführung für Frauenschulen und Fortbildungsschulen, Ausgabe B, Berlin/Leipzig, Verlag Teubner, 1922, $200 \mathrm{p}$.

Margarete Treuge, Einführung in die Bürgerkunde, ein Leitfaden für den staatsbürgerlichen Unterricht, Leipzig, Teubner, 1921 (1 ${ }^{\text {re }}$ éd.), 1922 (5éd.), 218 p.

\section{Fortbildungsschulen (cote SF-III)}

Prof. Fr. EHRINGHAus, Bürgerkunde und Volkswirtschaftslehre für Frauen, ein übersichtliches Lehr- und Lernbuch für Frauen, Frauenschulen, Töchterheime und ähnliche Anstalten, Göttingen, Vandenhoeck \& Ruprecht, 1922 ( $3^{\mathrm{e}}$ éd.), 1926 ( $5^{\mathrm{e}}$ éd.), $92 \mathrm{p}$.

W. Fender (Gewerbelehrer in Berlin), Richtlinien zu Vorbereitungen für den Unterricht in Gemeinschaftskunde für die Arbeiterschulen der Stadtgemeinde Berlin, 3. Schuljahr, Berlin, Ferdinand Ashelm, 1923, 181 p.

Otto Kinkel (Hauptlehrer in Lauterbach in Hessen), Neue Bürgerkunde für die Fortbildungsschulen des Freistaates Hessen, Heft 1: Die Verfassung des deutschen Reiches von 1919, in gemeinverständlicher Darstellung für das deutsche Volk und die Fortbildungsschulen, Gießen, Verlag von Emil Roth, 1919 ( $2^{\mathrm{e}}$ éd.), 40 p.

Michael Litschgy, Was mußt du wissen? Lesebuch für ländliche und gemischte Knabenfortbildungsschulen, Erster Teil: Der Mensch als Staatsbürger, Wittenberg, Herrosé's Verlag, 1924 (4 éd.), 1925 (5éd.), 1926 (6éd), 1928 (7éd.), 176 p.

H. Mohrenstecher (Fortbildungsschuldirektor, Landkreis Solingen), Lebens- und Bürger-

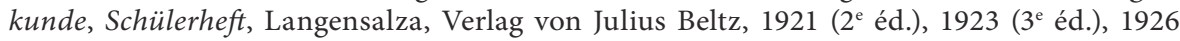
( $5^{\mathrm{e}}$ et $6^{\mathrm{e}}$ éd.), 1927 ( $7^{\mathrm{e}}$ éd.), 1930 (10éd.), 101 p.

Albrecht Niebuhr (Lehrer in Heiligentahl), Wichtige Wissensstoffe für die Landjugend. Sachlese und Lernbuch für die ländliche Fortbildungsschulen, 3. Teil, Lüneburg, Verlag von Herold und Mahlstab, 1928, 120 p.

Dr. Ing. Oskar Wende (Oberlehrer an der staatlichen Baugewerkschule Neukölln), Leitfaden der Staatsbürgerkunde für technische Fachschulen und andere Lehranstalten, Berlin, Verlag von Reuther und Reichard, 1919 ( $2^{\mathrm{e}}$ éd.), 1920 (3éd.), 160 p.

Robert Weselmeyer, Berufs- und Bürgerkunde für den Gebrauch an Berufs- und Fachschulen, 1. Teil (Hirt's Berufsschulswerk), Breslau, Ferdinand Hirt, 1930, 116 p.

2. Teil: Mittel und Oberstufe der Berufsschule, 1932, $136 \mathrm{p}$.

Deutsche Reichsbahngesellschaft (Lehrstoffhefte für Dienstanfängerschule), Staats- und Verwaltungskunde, Berlin, Verlag der Verkehrswissenschaftlichen Lehrmittelgesellschaft bei der Deutschen Reichsbahn, 1929, 1930 (2éd.), 34 p. 


\section{Zusammenfassung}

Anhand der Untersuchung von etwa 60 in der Weimarer Republik herausgegebenen Geschichts- und Staatsbürgerkundelehrbüchern analysiert der Beitrag die von diesen Schulbüchern verbreiteten Staatskonzepte und unterstreicht die Bedeutung des in ihrem Diskurs hergestellten Gegensatzes zwischen Verfassungsordnung und revolutionärer Unordnung sowie des Themas der Staatskontinuität über die revolutionäre Zäsur und den Regimewechsel von 1918 hinaus. Untersucht wird auch die Art und Weise, wie in den Lehrbüchern die demokratischen Ideen und das parlamentarische System den Schülern vermittelt werden. Dabei stellt sich also die Frage nach der schwierigen Anpassung einer ganzen sozialen Schicht von Lehrern und Juristen an die Demokratie.

\section{Résumé}

Sur la base du dépouillement d'une soixantaine de manuels scolaires d'histoire et d'éducation civique édités sous la République de Weimar, la contribution analyse les représentations de l'État véhiculées par ceux-ci et montre l'importance qu'ont eue, dans leur discours, l'opposition établie entre "l'ordre " constitutionnel et le "désordre " révolutionnaire, le thème de la continuité de l'État par-delà la césure révolutionnaire et le changement de régime de 1918. Elle aborde aussi la façon dont les idées démocratiques et le parlementarisme y sont expliqués aux élèves. C'est donc tout le problème de l'adaptation problématique à la démocratie d'une couche sociale de professeurs et de juristes qui est ici posé. 